\title{
A Low-power Bias Drift Cancellation Circuit Used For MEMS Gyroscopes
}

\author{
Risheng Liu ${ }^{1,}$ a , Qin Shi ${ }^{1, ~ b}$, Anping Qiu', Guoming Xia ${ }^{1}$, Yazhou Wang ${ }^{1}$, Yang Zhao 1,*, Dan \\ Zhang', Jing Wang ${ }^{3}$ \\ ${ }^{1}$ School of Mechanical Engineering, Nanjing University of Science and Technology, Nanjing 210094, \\ China. \\ ${ }^{2}$ Shanghai Aerospace Control Technology Institute, Shanghai 221116, China. \\ ${ }^{3}$ Sichuan Engineering Technical College, Deyang 618000, China. \\ rishliu@qq.com, ${ }^{b}$ sqinhy@njust.edu.cn \\ *Corresponding author: zhaoyang0216@yeah.net
}

Keywords: bias drift, low-power circuit, MEMS gyroscopes.

Abstract: This paper demonstrates a low-power oscillating circuit for MEMS gyroscopes which employs a chopper in driving-loop to realize bias drift cancellation. As a result of the misalignment of comb fingers inside microgyroscopes, driving force may give rise to a sensing-axis component which leads to the bias drift. By periodically chopping the driving-loop and sampling the bias when driving loop is open, sense mode output without drive-induced errors can be got. This method greatly improved the long-term stability of MEMS gyroscopes and the system power consumption is reduced to $95 \mathrm{~mW}$ from $768 \mathrm{~mW}$.

\section{Introduction}

MEMS gyroscope is an angular rate sensor, which is the core component of inertial navigation and attitude control systems. Compared with conventional gyroscopes, MEMS gyroscope has many advantages, such as small size, light weight, low power consumption, low cost, which make it widely used in industrial control, consumer electronics and military applications [1]. For over two decades of research, the performance of microgyroscopes has been improved greatly, but some defects of environment adaptability and long-term stability exposed under engineering application.

Bias Stability, a critical parameter for gyro performance, is defined as how much deviation or drift the sensor has from its mean value of the output rate. Essentially, the bias stability measurement shows how stable the bias of a gyro is over a certain specified period of time. The main factors that influence the bias stability are temperature and stress [2]. State-of-the-art work use temperature compensation to minimize the temperature error [3, 4]. However, few microgyroscopes employ stress compensation. Previous work found that the unequal gaps of comb fingers inside gyro arisen from fabrication error and adverse effects of stress will lead to bias drift [5]. This error term is in-phase with the useful signals and will become the main error source after the quadrature error being eliminated by phase sensitive demodulation.

At present, the research about bias drift cancellation is mainly focused on asymmetrical damping and misalignment of drive forcers. The Boeing Company presents several advanced error 
compensation and bias self-calibration techniques based on an extended gyro error model [6]. A continuous-time mode-reversal technique demonstrates an Allan-deviation improvement from $9^{\circ} / \mathrm{h}$ to $2^{\circ} / \mathrm{h}$ on a $3.3 \mathrm{kHz}$ vibratory gyroscope [7]. Analog Devices Inc. reported two self-calibration researches in 2014 and 2015 for drift cancellation by periodically reversing polarity of gyro's forcing voltage [8,9]. In order to recover the mitigate noise and bandwidth limitations caused by mode-reversal they presented a Dual Ramp Fusion technique which has enabled a five-fold reduction of the output drift.

Method of drive-induced error cancellation circuit is proposed in this work. The implementation is based on z-axis silicon microgyroscopes (Fig.1.) with a low power circuit driven by amplitude control closed-loop and detected by open-loop. When the drive loop is cut off, drive-induced error also disappears while the useful output signals will not because of the high quality factor. Then we use software algorithms to sample the output with the absence of drive-induced error. By means of periodically switching the drive force on and off, bias drift will no longer be affected by stress and allows for stability improvement.

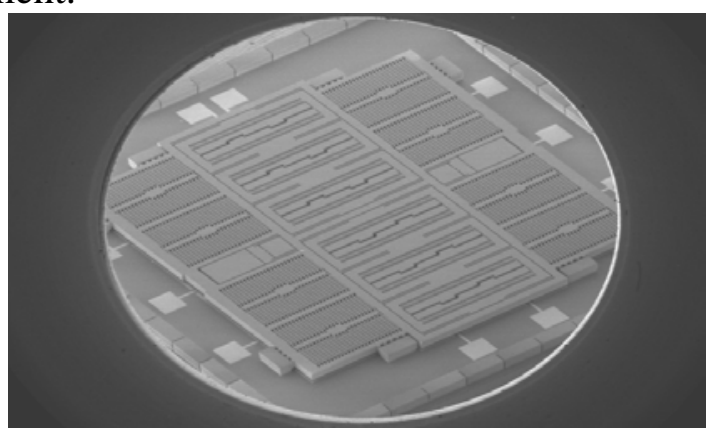

Fig. 1 Stereoscan photograph of dual-mass silicon microgyroscope designed by our group

\section{Drive-induced Error}

Bias (zero rate output) is the average over a specified time of gyro output measured at specified operating conditions that has no correlation with input rotation [10]. Bias errors can result from multiple sources including misalignment of the drive forcers, asymmetrical damping, and electrical feed-through from the drive element to the sense element. Since the quality factor of drive-mode is high enough (up to 80,000) so that error from asymmetrical damping is negligible. Therefore the drive-induced error is mainly from misalignment of the drive forcers because electrical feed-through is extremely small compared with the former. The principle of error's emergence is shown in Fig.2.

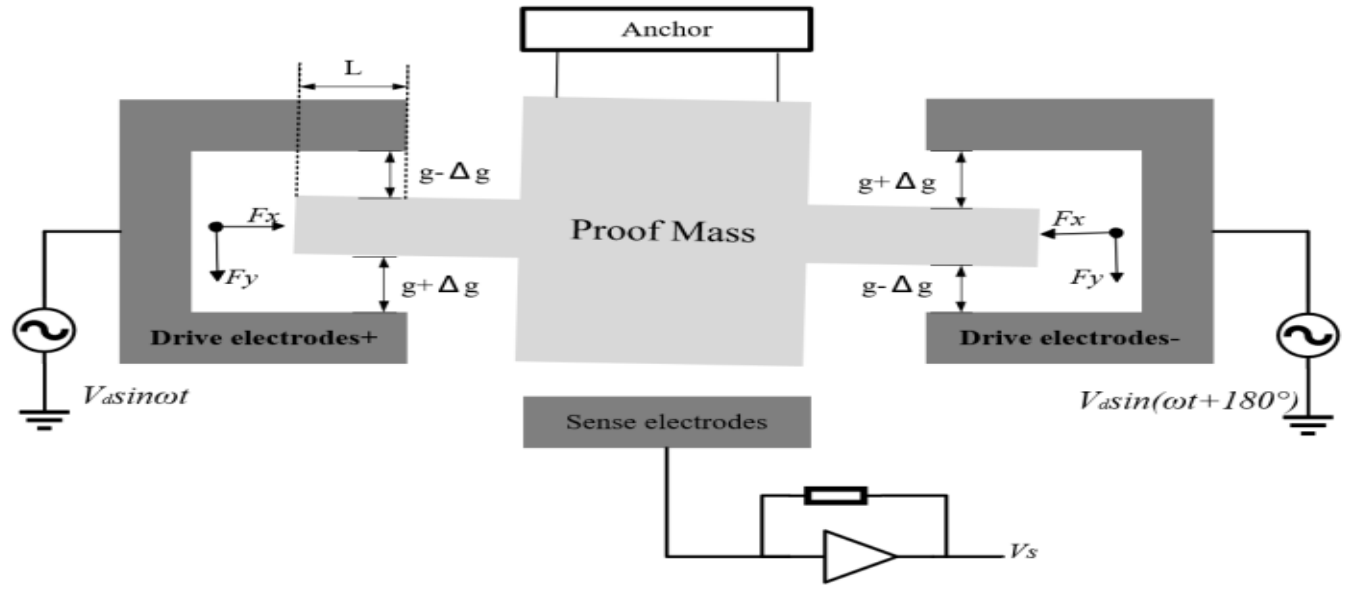

Fig. 2 Sketch map of drive-induced error generating mechanism 
When the gap width of comb is different with a misalignment angle of $\alpha$, the resultant force in sensing direction will not be zero, resulting in a y-axis component:

$$
F_{y}=F_{x} \sin \alpha \approx F_{x} \alpha
$$

We use the framework developed in [5], the coupling coefficient between drive and sense element is:

$$
\alpha=\frac{\mathrm{F}_{\mathrm{x}}}{\mathrm{F}_{\mathrm{y}}}=\frac{\mathrm{g}^{2}}{2 \Delta \mathrm{gL}}
$$

Where $g$ and $\Delta \mathrm{g}$ are the nominal and deviation value of gap width of comb, $\mathrm{L}$ is the overlap between fixed and mobile comb fingers. We can also come up with the equation of in-phase error when there is an angular rate input [5]:

$$
\Omega_{Y}=\frac{\omega}{Q_{X}} \frac{\Delta g L}{g^{2}} \frac{180}{\pi}
$$

In which $\omega$ is the drive frequency and $Q_{X}$ is the quality factor. So $\alpha$ is only related to the size of comb, and we can find out that by improving fabrication process, decrease overlap area, greaten gap width and quality factor the drive-induced error will be reduced. But the comb capacitor changes along with the misalignment of gap due to stress, which is difficult to obtain directly when the gyro is running, so this error term can hardly be eliminated by compensating on-line.

\section{Low-power Oscillating Circuit}

The system overview is shown in Fig.3. The gyroscope is driven by closed-loop self-oscillation circuit and realizes amplitude control through automatic gain control (AGC) amplifier. Together with open-loop detection circuit, the gyro measurement and control system is constructed. The preamplifier converts current from driving and sensing electrodes voltage with gain. A variable gain amplifier AD8338 from Analog Devices, Inc. [11] is used to configure as an AGC Amplifier (Fig.4) which completely replaces the conventional AGC loop consists of full wave rectifier, low pass filter and PI controller, and make the circuit more compact and lower power consumption. More than that, all other components are selected to accommodate the $0-5 \mathrm{~V}$ low power supply voltage. Then we utilize an analog switch to chop the driving loop periodically and drive the vibration of resonator.

The lower right in Fig.3 is the output algorithm based on LabVIEW. In order to solve the drive-induced error caused by comb gap deviation, square wave is generated to trigger the analog switch on and off in drive circuits. During the very short time of ring-down when driving force is removed, the amplitude will not decay to zero as a result of high quality factor of drive mode while the output signal contains no drive-induced error, as shown in Fig.5. So each section of output signal without drive force will be sampled so that drive-induced error cancellation can be realized. The AC signals will be low-pass filtered after multiplication demodulation and we can get the output voltage proportional to angular rate input triggered by the square wave.

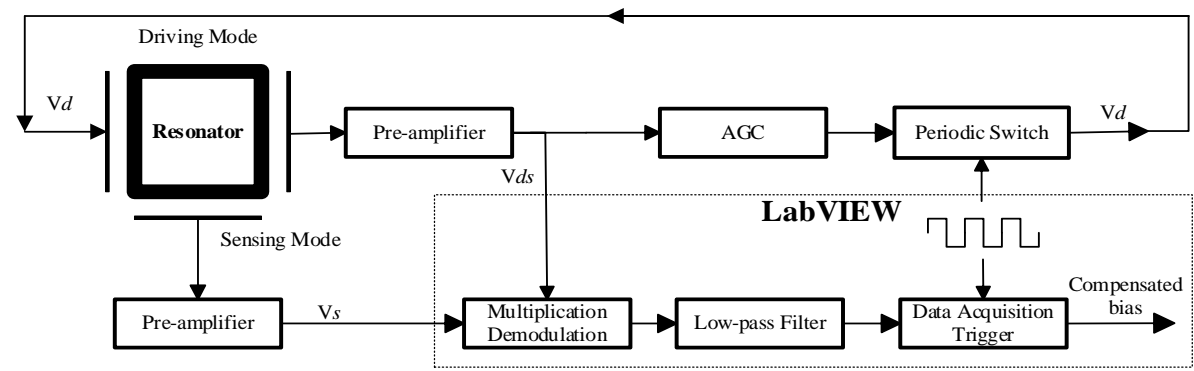

Fig. 3 Framework of the whole system 

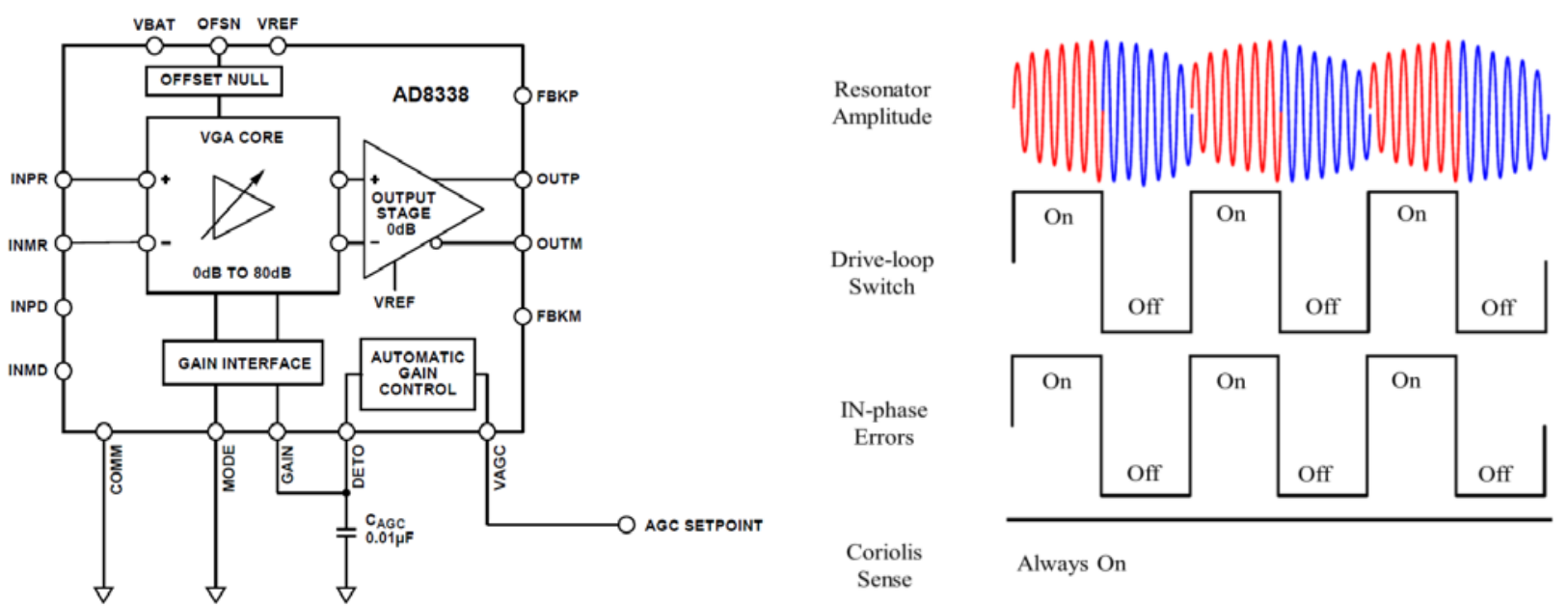

Always On

Fig. 4 AD8338 configured as an AGC amplifier Fig. 5 Principle of drive-induced error cancellation

\section{Experiment Validation}

At first we verified that there is no dive-induced error when the drive loop is open as shown in Fig.6. We can find a drop in the bias drift of gyroscope which means the disappearance of error term. After that output signal began to fall along with the amplitude of resonator, hence we have to make it stable by data fitting. During the continuous work of the whole system, we collect all the functional signals as shown in Fig.7. There is significantly reduced bias when the resonance oscillation falling without driving voltage. When the system is operating normally with the previous driving loop, we put some weights on gyro to disclose the bad influence of stress compared with the calibrated one, results are shown in Fig.8. it is verified that the applied stress will change the gap between comb fingers and affect the bias drift, the greater the stress applied, the greater the bias drift. Finally we get the results under the condition of self-calibration that the extra stress will not exert an impact. Meanwhile, the power supply voltage of the previous circuit is $\pm 12 \mathrm{~V}$ and working current is $32 \mathrm{~mA}$, compared with the low-power circuit of $5 \mathrm{~V}$ and $19 \mathrm{~mA}$, it has enabled an eight-fold reduction of power consumption from $768 \mathrm{~mW}$ to $95 \mathrm{~mW}$.
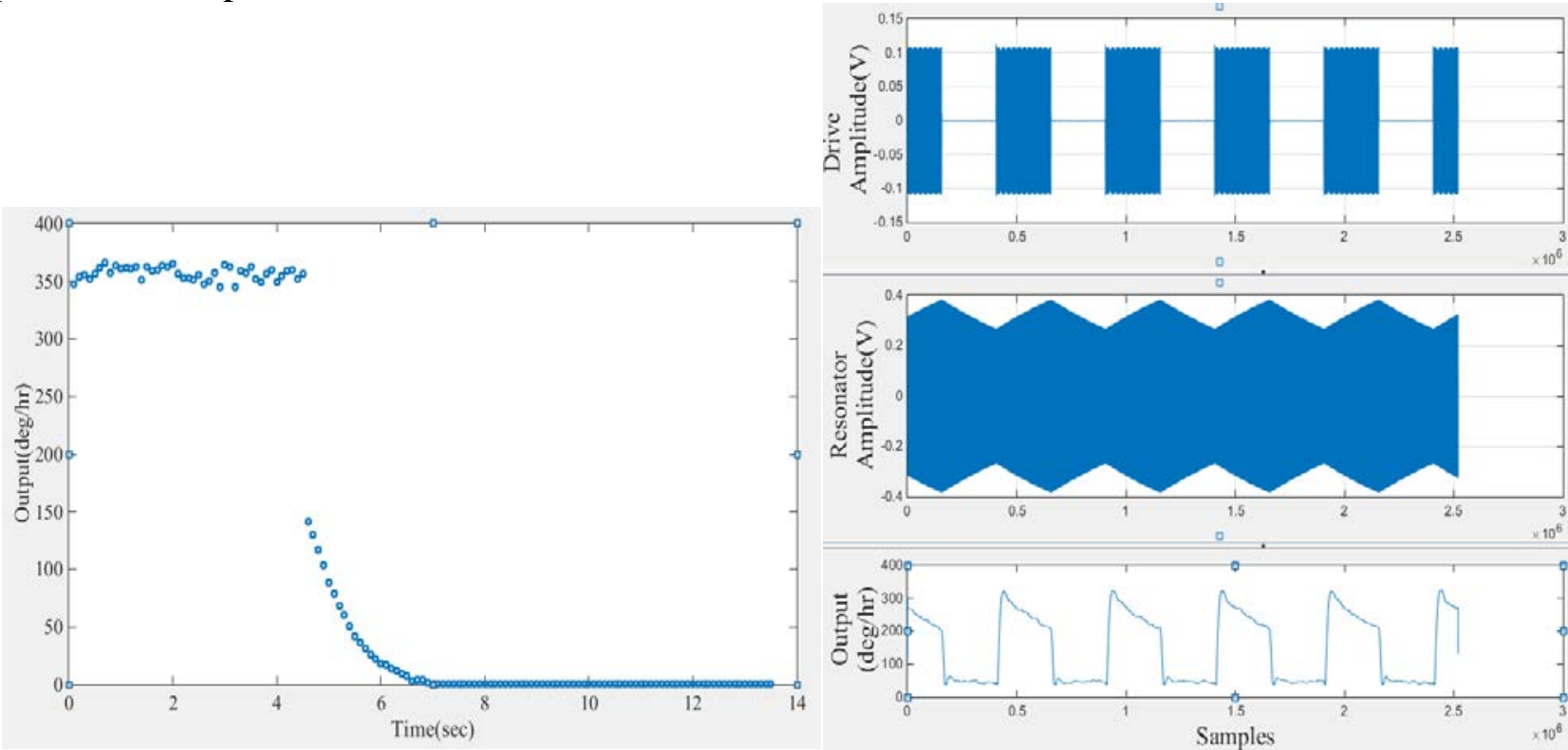

Fig. 6 Bias drop when drive force is cut off

Fig.7 Some vital parameters acquisitioned by DAQ 

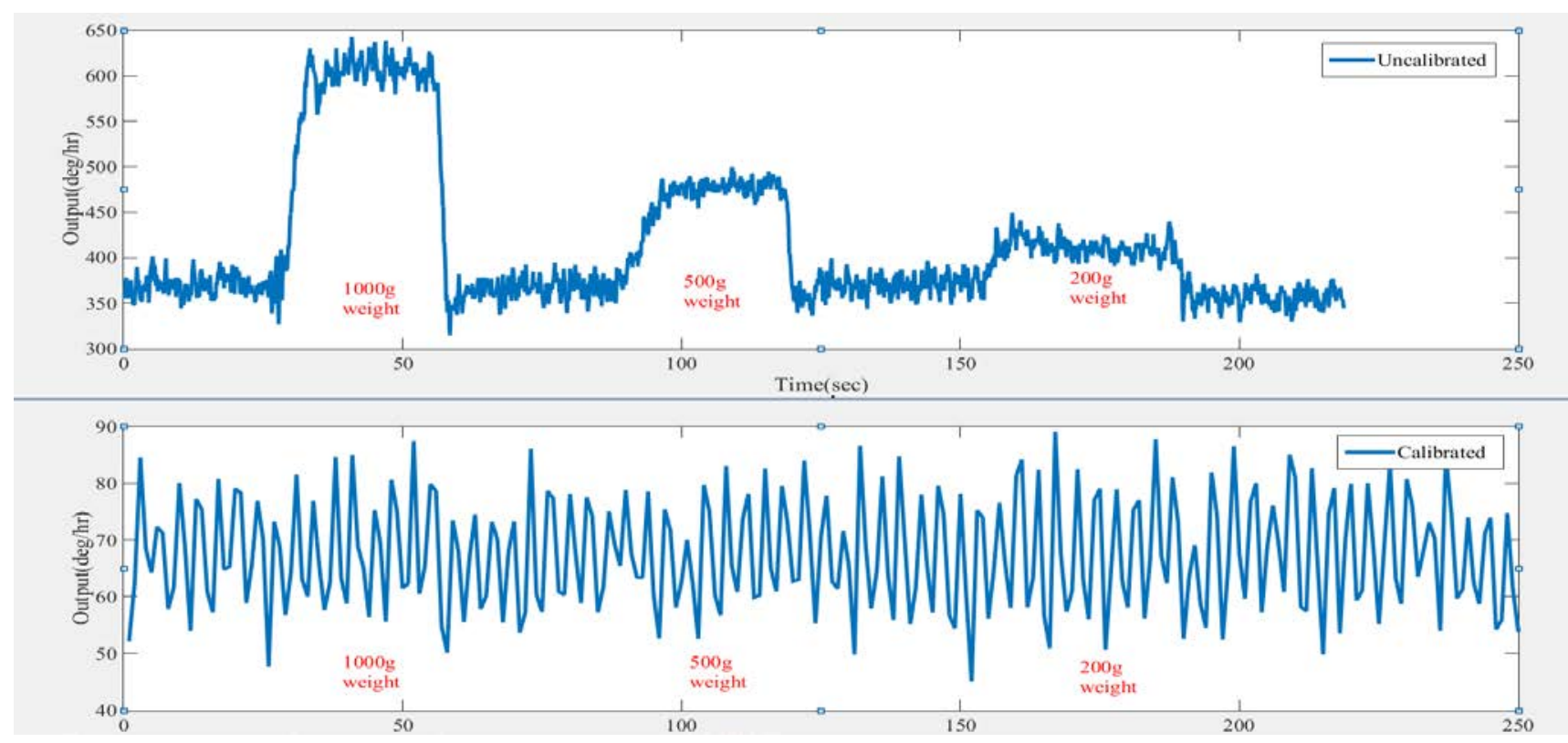

Fig. 8 Gyro’s output under different stress

\section{Conclusions}

This paper provides a bias drift cancellation circuit to eliminate the drive-induced error. The method provides an effective way to improve stability and reliability of microgyroscopes under temperature, stress and long-term service conditions. In future works, we plan to utilize multiple gyroscopes of the same type simultaneously or only one gyroscope using information fusion technology based on signal filter algorithm to realize continuous detecting.

\section{Acknowledgments}

This work was supported by the Fundamental Research Funds for the Central Universities, No. 309171 B8810.

\section{References}

[1] Z. S. Ma. Design of the Semi-phisical Simulation System of MEMS Gyroscope Based on LabVIEW. Ph. M. Dissertation. Nanjing University of Science \& Technology, Dec. 2017. (in Chinese)

[2] E. Tatar, T. Mukherjee and G. K. Fedder, "Stress Effects and Compensation of Bias Drift in a MEMS Vibratory-Rate Gyroscope," in Journal of Microelectromechanical Systems, vol. 26, no. 3, pp. 569-579, June 2017.

[3] A. D. Challoner, H. H. Ge, and J. Y. Liu, "Boeing disc resonator gyroscope," in Proc. PLANS, Monterey, CA, USA, May 2014, pp. 504-514.

[4] I. P. Prikhodko, A. A. Trusov, and A. M. Shkel, "Compensation of drifts in high-Q MEMS gyroscopes using temperature self-sensing," Sens. Actuators A, Phys., vol. 201, pp. 517-524, Oct. 2013.

[5] Q. Shi, A.P. Qiu, Y. SU, X.H. Zhu, "Study on the Effects of Machining Error on the Z-Axis Silicon Microgyroscope," Chinese Journal of Sensors and Actuators, vol. 19, Oct. 2006, pp. 2182-2185.(in Chinese)

[6] H. H. Ge, J. Y. Liu and B. Buchanan, "Bias self-calibration techniques using silicon disc resonator gyroscope," 2015 IEEE International Symposium on Inertial Sensors and Systems (ISISS) Proceedings, Hapuna Beach, HI, 2015, pp. 1-4.

[7] M. H. Kline et al., "MEMS gyroscope bias drift cancellation using continuous-time mode reversal," 2013 Transducers \& Eurosensors XXVII: The 17th International Conference on Solid-State Sensors, Actuators and Microsystems (TRANSDUCERS \& EUROSENSORS XXVII), Barcelona, 2013, pp. 1855-1858.

[8] I. P. Prikhodko et al., "In-run bias self-calibration for low-cost MEMS vibratory gyroscopes," 2014 IEEE/ION Position, Location and Navigation Symposium - PLANS 2014, Monterey, CA, 2014, pp. 515-518.

[9] I. P. Prikhodko et al., "Continuous self-calibration canceling drive-induced errors in MEMS vibratory gyroscopes," 
2015 Transducers - 2015 18th International Conference on Solid-State Sensors, Actuators and Microsystems (TRANSDUCERS), Anchorage, AK, 2015, pp. 35-38.

[10] C. Acar, A. Shkel, MEMS Vibratory Gyroscopes Structural Approaches to Improve Robustness. Springer US, 2009.

[11] Analog Devices, Inc. Low Power, $18 \mathrm{MHz}$ Variable Gain Amplifier AD8338 Data Sheet. Last Content Update: 02/23/2017. www.analog.com. 